\title{
Superluminary Universe: A Possible Solution to the Initial Value Problem in Cosmology
}

\author{
J. W. Moffat \\ Department of Physics, University of Toronto, Toronto, Ontario M5S 1A7, Canada
}

\begin{abstract}
The spontaneous breaking of local Lorentz invariance in the early Universe, associated with a first order phase transition at a critical time $t_{c}$, generates a large increase in the speed of light and a superluminary communication of information occurs, allowing all regions in the Universe to be causally connected. This solves the horizon problem, leads to a mechanism of monopole suppression in cosmology and can resolve the flatness problem. After the critical time $t_{c}$, local Lorentz (and diffeomorphism) invariance is restored and light travels at its presently measured speed. The kinematical and dynamical aspects of the generation of quantum fluctuations in the superluminary Universe are investigated. A scale invariant prediction for the fluctuation density amplitude is obtained.
\end{abstract}

Published in: International Journal of Modern Physics D, Vol. 2, No. 3 (1993) 351-365.

\section{UTPT-92-14. e-mail: moffat@medb.physics.utoronto.ca}

\section{Introduction}

In order to solve the problem of the initial value of the Universe, popularly expressed in terms of the standard three problems: the horizon problem, the flatness problem and the monopole or relic particle suppression problem, the idea of an inflationary Universe was introduced[1], 2]. By allowing for an exponential growth of the very early Universe, caused by a huge vacuum energy generating a de Sitter Universe phase, these initial value problems could be solved. In addition, the inflationary mechanism was able to predict the existence of quantum fluctuations which could be the seeds of Galaxy formation. However, the inflationary scenario ran into difficulties already in the first paper by Guth, since in order to have sufficient inflation, it is necessary to keep the "false" vacuum reasonably stable, leading to a small bubble nucleation rate and to the lack of bubble coalescence. The final configuration of 
the Universe is very inhomogeneous in disagreement with the observations. This led to modified models introducing special forms for the Higgs potential $V(\phi)$ with an extremely flat $V(\phi)$ for $\phi \leq \sigma\left(\sigma=<\phi>_{0}\right)[2]$. These models used weakly first order or even second order phase transitions. Instead of many bubbles colliding and having to coalesce, the models have one huge bubble containing everything observable in the Universe now. A serious problem with this kind of model is that the density perturbations, $\delta \rho / \rho$, are too large by a factor of order $10^{6}$, unless the parameter in the potential (e.g. Coleman-Weinberg) is artificially reduced by a factor $\sim 10^{12}$.

In Linde's chaotic inflation[2], which does not involve any phase transitions, the potential has the simple form: $V(\phi)=\lambda \phi^{4}$. Assuming the slow motion of $\phi$ from some initial value $\phi_{0}$ towards the minimum, one can obtain sufficient inflation. The main difficulties are: 1) To obtain observationally acceptable values for the density perturbations, it is necessary to fine-tune $\lambda$ to very small values: $\lambda \approx 10^{-12}-10^{-14}$. There is no obvious physical reason why the coupling constant $\lambda$ should be so small. 2) It must be assumed that the kinetic energy of the scalar field is small compared to its potential energy, which leads to the condition that the field be uniform over sizes bigger than the Hubble radius, which is not in keeping with the original ideas of inflation.

Other scenarios include models based on the Brans-Dicke theory of gravity (extended inflation and hyper-extended inflation) and extensions of this theory [2]. These models also have their problems e.g. by introducing unacceptable distortions of the microwave background. Up till the present time, there appears to be no satisfactory model of inflation.

In the following, we shall consider a new scenario which is capable of solving the horizon, excess relic particle and flatness problems and leads to predictions for small scale inhomogeneities. In previous work [3], the local Lorentz and diffeomorphism symmetries of Einstein's gravitational theory are spontaneously broken by a Higgs mechanism in the early Universe, with the symmetry breaking pattern: $S O(3,1) \rightarrow$ $O(3)$, at a critical temperature $T_{c}$, below which the symmetry is restored.

Within this symmetry breaking scheme, the super-Hamiltonian constraint for the wave function, $H_{0} \Psi=0$, was relaxed, leading to a time dependent Schrödinger equation. In this framework, quantum mechanics and gravitation were united in a meaningful observational scheme. The local Lorentz invariant structure of the gravitational Lagrangian is maintained as a "hidden" symmetry. After the spacetime symmetries are restored in the early Universe for a temperature $T<T_{c}$, the wave function has an oscillatory behavior, and is peaked about a set of classical Lorentzian four-geometries. One may then use the WKB approximation and the tangent vector to the configuration space - for paths about which $\Psi$ is peaked - to define the proper time $\tau$ along the classical trajectories [4]. Thus, once the mechanism of spontaneous breaking of the spacetime symmetries has taken place in the early Universe, then the problems of time and time's arrow are solved by means of the classical HamiltonJacobi equation and the classical trajectories define a time and time's direction in the symmetric phase. The Universe is then clearly divided into a quantum gravity 
regime and a classical regime, making the WKB solution to the origin of the time variable unambiguous without arbitrary boundary conditions.

In the following, we shall investigate further the physical consequences of the spontaneous breaking of the symmetries of spacetime in the early Universe. We shall postulate that the velocity of light goes through a first order phase transition at a critical time $t \sim t_{c}$, associated with the spontaneous Higgs breaking of local Lorentz invariance. This defines a "superluminary" phase of the early Universe, which solves the horizon problem, the flatness problem and suppresses any relic magnetic monopoles. In Section 4, the generation of quantum fluctuations in the superluminary Universe is studied, and a scale invariant gaussian fluctuation density amplitude is derived from a model of the the critical phase transition.

\section{Spontaneous Breaking of Spacetime Symmetries}

Let us define the vierbein $e_{\mu}^{a}$ in terms of the metric:

$$
g_{\mu \nu}(x)=e_{\mu}^{a}(x) e_{\nu}^{b}(x) \eta_{a b} .
$$

The vierbeins $e_{\mu}^{a}$ satisfy the orthogonality relations:

$$
e_{\mu}^{a} e_{b}^{\mu}=\delta_{b}^{a}, \quad e_{a}^{\mu} e_{\nu}^{a}=\delta_{\nu}^{\mu},
$$

which allow us to pass from the flat tangent space coordinates (the fibre bundle tangent space) labeled by $a, b, c \ldots$ to the the world spacetime coordinates (manifold) labeled by $\mu, \nu, \rho \ldots$. The fundamental form (1) is invariant under Lorentz transformations:

$$
e_{\mu}^{\prime a}(x)=L_{b}^{a}(x) e_{\mu}^{b}(x),
$$

where $L_{b}^{a}(x)$ are the homogeneous $S O(3,1)$ Lorentz transformation coefficients that can depend on position in spacetime, and which satisfy

$$
L_{a c}(x) L_{d}^{a}(x)=\eta_{c d}
$$

The $e_{\mu}^{a}$ obey the equation:

$$
D_{\sigma} e_{\mu}^{a}=e_{\mu, \sigma}^{a}+\left(\Omega_{\sigma}\right)_{c}^{a} e_{\mu}^{c},
$$

where $D_{\sigma}$ is the covariant derivative operator with respect to the (spin) gauge connection $\Omega_{\mu}$.

In holonomic coordinates, the curvature tensor is given by

$$
R_{\sigma \mu \nu}^{\lambda}=\left(R_{\mu \nu}\right)_{b}^{a} e_{a}^{\lambda} e_{\sigma}^{b}
$$

and the scalar curvature takes the form

$$
R=e^{\mu a} e^{\nu b}\left(R_{\mu \nu}\right)_{a b} .
$$


The action for Einstein gravity is written as

$$
S_{G}=-\frac{c^{4}}{16 \pi G} \int d^{4} x e(R+2 \Lambda)
$$

where $e \equiv \sqrt{-g}=\operatorname{det}\left(e_{\mu}^{a} e_{a \nu}\right)^{1 / 2}$ and $\Lambda$ is the cosmological constant.

We shall consider a specific kind of symmetry breaking in the early Universe, in which the local Lorentz vacuum symmetry is spontaneously broken by a Higgs mechanism. We postulate the existence of four scalar fields, $\phi_{a}$, and assume that the vacuum expectation value (vev) of the scalar fields, $\left\langle\phi_{a}\right\rangle_{0}$, will vanish for some temperature $\mathrm{T}$ less than a critical temperature $T_{c}$, when the local Lorentz symmetry is restored. Above $T_{c}$ the non-zero vev will break the symmetry of the gound state of the Universe from $S O(3,1)$ down to $O(3)$. The domain formed by the direction of the vev of the Higgs field will produce a time arrow pointing in the direction of increasing entropy and the expansion of the Universe. The four real scalar fields $\phi^{a}(x)$ are invariant under Lorentz transformations [3]:

$$
\phi^{\prime a}(x)=L_{b}^{a}(x) \phi^{b}(x)
$$

We can use the vierbein to convert $\phi^{a}$ into a 4 -vector in coordinate space: $\phi^{\mu}=e_{a}^{\mu} \phi^{a}$. The covariant derivative operator acting on $\phi^{a}$ is defined by

$$
D_{\mu} \phi^{a}=\left[\partial_{\mu} \delta_{b}^{a}+\left(\Omega_{\mu}\right)_{b}^{a}\right] \phi^{b} .
$$

We shall now introduce a Higgs sector into the Lagrangian density such that the gravitational vacuum symmetry, which we set equal to the Lagrangian symmetry at low temperatures, will break to a smaller symmetry at high temperature. The pattern of vacuum phase transition that emerges contains a symmetry antirestoration[5-12]. This vacuum symmetry breaking leads to the interesting possibility that exact zero temperature conservation laws e.g. electric charge and baryon number are broken in the early Universe. In our case, we shall find that the spontaneous breaking of the Lorentz symmetry of the vacuum leads to a violation of the exact zero temperature conservation of energy in the early Universe.

Let us consider the Lorentz invariant Higgs potential:

$$
V(\phi)=-\frac{1}{2} \mu^{2} \sum_{a=0}^{3} \phi_{a} \phi^{a}+\lambda \sum_{a=0}^{3}\left(\phi_{a} \phi^{a}\right)^{2},
$$

where we choose $\lambda>0$, so that the potential is bounded from below. Our Lagrangian density now takes the form:

$$
\mathcal{L}=\mathcal{L}_{G}+\sqrt{-g}\left[\frac{1}{2} D_{\mu} \phi_{a} D^{\mu} \phi^{a}-V(\phi)\right] .
$$

If $V$ has a minimum at $\phi_{a}=v_{a}$, then the spontaneously broken solution is given by $v_{a}^{2}=\mu^{2} / 4 \lambda$ and an expansion of $V$ around the minimum yields the mass matrix:

$$
\left(\mu^{2}\right)_{a b}=\frac{1}{2}\left(\frac{\partial^{2} V}{\partial \phi_{a} \partial \phi_{b}}\right)_{\phi_{a}=v_{a}} .
$$


We can choose $\phi_{a}$ to be of the form

$$
\phi_{a}=\left(\begin{array}{c}
0 \\
0 \\
0 \\
v
\end{array}\right)=\delta_{a 0}\left(\mu^{2} / 4 \lambda\right)^{1 / 2}
$$

All the other solutions of $\phi_{a}$ are related to this one by a Lorentz transformation. Then, the homogeneous Lorentz group $S O(3,1)$ is broken down to the spatial rotation group $O(3)$. The three rotation generators $J_{i}(i=1,2,3)$ leave the vacuum invariant

$$
J_{i} v_{i}=0
$$

while the three Lorentz-boost generators $K_{i}$ break the vacuum symmetry

$$
K_{i} v_{i} \neq 0
$$

The $J_{i}$ and $K_{i}$ satisfy the usual commutation relations

$$
\left[J_{i}, J_{j}\right]=i \epsilon_{i j k} J_{k}, \quad\left[J_{i}, K_{j}\right]=i \epsilon_{i j k} K_{k}, \quad\left[K_{i}, K_{j}\right]=-i \epsilon_{i j k} K_{k} .
$$

The mass matrix $\left(\mu^{2}\right)_{a b}$ can be calculated from (13):

$$
\left(\mu^{2}\right)_{a b}=\left(-\frac{1}{2} \mu^{2}+2 \lambda v^{2}\right) \delta_{a b}+4 \lambda v_{a} v_{b}=\mu^{2} \delta_{a 0} \delta_{b 0}
$$

where $v$ denotes the magnitude of $v_{a}$. There are three zero-mass Goldstone bosons, the same as the number of massive vector bosons, and there are three massless vector bosons corresponding to the unbroken $O(3)$ symmetry. In addition to these particles, one massive physical boson particle $h$ remains, after the spontaneous breaking of the vacuum. The vector boson mass term is given in the Lagrangian by

$$
\mathcal{L}_{\Omega}=\frac{1}{2} \sqrt{-g}\left(\Omega_{\mu}\right)^{a b} v_{b}\left(\Omega^{\mu}\right)_{a}^{c} v_{c}=\frac{1}{2} \sqrt{-g} \sum_{i=1}^{3}\left(\left(\Omega_{\mu}\right)^{i 0}\right)^{2}\left(\mu^{2} / 4 \lambda\right) .
$$

A phase transition is assumed to occur at the critical temperature $T_{c}$, when $v_{a} \neq 0$ and the Lorentz symmetry is broken and the three gauge fields $\left(\Omega_{\mu}\right)^{i 0}$ become massive vector bosons. Below $T_{c}$ the Lorentz symmetry is restored, and we regain the usual classical gravitational field with massless gauge fields $\Omega_{\mu}$. The symmetry breaking will extend to the singularity or the possible singularity-free initial state of the big bang, and since quantum effects associated with gravity do not become important before $T \sim 10^{19} \mathrm{GeV}$, we expect that $T_{c} \leq 10^{19} \mathrm{GeV}$.

In most known cases of phase transitions of the first and second kind, the more symmetrical phase corresponds to higher temperatures and the less symmetrical one to lower temperatures. A transition from an ordered to a disordered state usually occurs with increasing temperature. Examples of two known exceptions in Nature are the "lower Curie point" of Rochelle salt, below which the crystal is orthorhombic, 
but above which it is monoclinic. Another example is the gapless superconductor. A calculation of the effective potential for the Higgs breaking contribution in (12) shows that extra minima in the potential $V(\phi)$ can occur for a noncompact group such as $S O(3,1)[12$.

The entropy will rapidly increase during the phase transition, when the symmetry is restored, and for a closed Universe will reach a maximum at the final singularity, provided that no further phase transition occurs which breaks the Lorentz symmetry of the vacuum. Thus, the symmetry breaking mechanism explains in a natural way the low entropy at the initial singularity and the large entropy at the final singularity. Since the ordered phase is at a much lower entropy than the disordered phase and due to the existence of a domain determined by the direction of the vev of the fields, $<\phi_{a}>_{0}$, a natural explanation is given for the cosmological arrow of time and the origin of the second law of thermodynamics. Thus, the spontaneous symmetry breaking of the gravitational vacuum corresponding to the breaking pattern, $S O(3,1) \rightarrow O(3)$, leads to a manifold with the structure $R \times O(3)$, in which time appears as an absolute external parameter. The vev of the fields, $<\phi_{a}>_{0}$, points in a chosen direction of time to break the symmetry creating an arrow of time.

The total action for the theory is

$$
S=S_{G}+S_{M}+S_{\phi}
$$

where $S_{G}$ is given by (8) and $S_{M}$ is the usual matter action for gravity. Moreover,

$$
S_{\phi}=\int d^{4} x \sqrt{-g}\left[\frac{1}{2}\left(D_{\mu} \phi_{a} D^{\mu} \phi^{a}-V(\phi)\right] .\right.
$$

Performing a variation of $S$ leads to the field equations:

$$
G^{\mu \nu} \equiv R^{\mu \nu}-\frac{1}{2} g^{\mu \nu} R=\frac{8 \pi G}{c^{4}}\left(T^{\mu \nu}+C^{\mu \nu}\right)+\Lambda g^{\mu \nu},
$$

where $T^{\mu \nu}$ is the energy-momentum tensor for matter. The energy-momentum tensor for the coordinate scalar $\phi_{a}$ fields is of the usual form:

$$
C^{\mu \nu}=D^{\mu} \phi_{a} D^{\nu} \phi^{a}-\mathcal{L}_{\phi} g^{\mu \nu}
$$

The Higgs mass and graviton mass contributions proportional to $v^{a}=<\phi^{a}>_{0}$ are given by (18) and (19). Since we assume that the symmetry breaking pattern is $S O(3,1) \rightarrow O(3)$, there will be three massless gauge vector fields $\left(\Omega_{\mu}\right)_{n m}=-\left(\Omega_{\mu}\right)_{m n}$, denoted by $U_{\mu}^{n}$, three massive vector bosons $\left(\Omega_{\mu}\right)_{0 i}=-\left(\Omega_{\mu}\right)_{i 0}$, denoted by $V_{\mu}^{i}$ and one massive boson $h$. Because $G^{\mu \nu}$ satisfies the Bianchi identities $G_{; \nu}^{\mu \nu}=0$, we find in the broken symmetry phase:

$$
T_{; \nu}^{\mu \nu}=K^{\mu},
$$


where ; denotes the covariant derivative with respect to the Christoffel symbols $\Gamma_{\mu \nu}^{\lambda}$, and $K^{\mu}$ contains the mass terms proportional to $v_{a}=<\phi_{a}>_{0}$. Thus, the conservation of energy-momentum is spontaneously violated and matter can be created in this broken symmetry phase. When the temperature passes below the critical temperature, $T_{c}$, then $v_{a}=0$ and the action is restored to its classical form with a symmetric degenerate vacuum and a massless spin gauge connection $\left(\Omega_{\mu}\right)_{b}^{a}$, and we regain the standard energy-momentum conservation laws: $T_{; \nu}^{\mu \nu}=0$.

\section{$3 \quad$ Field Equations in the Broken Symmetry Phase}

The "Newtonian" spacetime manifold in the broken phase has the symmetry $R \times$ $O(3)$. The three-dimensional space with $O(3)$ symmetry is assumed to be homogeneous and isotropic and yields the usual maximally symmetric three-dimensional space:

$$
d \sigma^{2}=R^{2}(t)\left[\frac{d r^{2}}{1-k r^{2}}+r^{2}\left(d \theta^{2}+\sin ^{2} \theta d \phi^{2}\right)\right]
$$

where $t$ is the external time variable. This is the Robertson-Walker theorem for our ordered phase of the vacuum and it has the correct subspace structure for the FRW Universe with the metric:

$$
d s^{2}=d t^{2} c^{2}-R^{2}(t)\left[\frac{d r^{2}}{1-k r^{2}}+r^{2}\left(d \theta^{2}+\sin ^{2} \theta d \phi^{2}\right)\right] .
$$

The null geodesics of the metric (26) are the light paths of the subspace and $d \lambda=$ $d s / c$ measures the time at each test particle [15], where $c$ is a constant with the dimensions of the speed of light.

In the broken symmetry phase, the "time" $t$ is the absolute physical time measured by standard clocks. In contrast to GR, while $\langle\phi\rangle$ is non-zero, we no longer have re-parameterization invariance and time is no longer an arbitrary label.

The total action for the theory in the broken symmetry phase, $T>T_{c}$, is

$$
S=S_{G}+S_{M}+S_{h}+S_{V}
$$

Let us consider small oscillations about the true minimum and define a shifted field:

$$
\phi_{a}^{\prime}=\phi_{a}-v_{a}
$$

After Faddeev-Popov ghost fixing, we can define a new set of extended BecchiRouet-Stora (BRS) 13 Lorentz gauge transformations under which $S$ is invariant, and a set of Ward-Takahashi identities can be found. Let us perform a Lorentz transformation on $\phi^{a}$, so that we obtain:

$$
\phi^{0}=h, \quad \phi^{1}=\phi^{2}=\phi^{3}=0 .
$$


In this special coordinate frame, the remaining component $h$ is the physical Higgs particle that survives after the Goldstone modes have been removed. This corresponds to choosing the "unitary gauge" in the standard electroweak model [14]. In our specially chosen coordinate frame in which (29) holds and the metric (26) is realized, we have

$$
S_{h}=\int d^{4} x \sqrt{-g}\left[\frac{1}{2} \partial_{\mu} h \partial^{\mu} h-V(h)\right]
$$

where

$$
V(h)=4 \lambda v^{2} h^{2}+4 \lambda v h^{3}+\lambda h^{4}-\frac{1}{2} V_{\mu}^{2} h^{2}-v V_{\mu}^{2} h,
$$

and we have for convenience suppressed the index $i$ on $V_{\mu}^{i}$. Moreover,

$$
S_{V}=\frac{1}{2} m^{2} \int d^{4} x \sqrt{-g} g^{\mu \nu} V_{\mu} V_{\nu}
$$

where the mass $m \propto<h>$.

The field equations are of the form:

$$
G^{\mu \nu} \equiv R^{\mu \nu}-\frac{1}{2} g^{\mu \nu} R=\frac{8 \pi G}{c^{4}}\left(T^{\mu \nu}+K^{\mu \nu}+H^{\mu \nu}\right)+\Lambda g^{\mu \nu},
$$

where $K^{\mu \nu}$ is given by

$$
K^{\mu \nu}=m^{2}\left(V^{\mu} V^{\nu}-\frac{1}{2} g^{\mu \nu} V^{\beta} V_{\beta}\right) .
$$

Moreover, the $h$ field energy-momentum tensor is of the usual form:

$$
H^{\mu \nu}=\partial^{\mu} h \partial^{\nu} h-\mathcal{L}_{h} g^{\mu \nu} .
$$

The spin connections $\left(\Omega_{\sigma}\right)^{a b}$ are determined by the equations:

$$
e_{\mu, \sigma}^{a}+\left(\Omega_{\sigma}\right)_{c}^{a} e_{\mu}^{c}-\Gamma_{\sigma \mu}^{\rho} e_{\rho}^{a}=0,
$$

which leads to the solution:

$$
\left(\Omega_{\sigma}\right)^{a b}=-e^{b \mu}\left(e_{\mu, \sigma}^{a}-e_{\rho}^{a} \Gamma_{\sigma \mu}^{\rho}\right) .
$$

For an FRW manifold, we have

$$
e_{\mu}^{0}=\delta_{\mu}^{0}, \quad e_{\mu}^{1}=\frac{R}{\left(1-k r^{2}\right)^{1 / 2}} \delta_{\mu}^{1}
$$

and

$$
e_{\mu}^{2}=R r \delta_{\mu}^{2}, \quad e_{\mu}^{3}=\operatorname{Rr} \sin \theta \delta_{\mu}^{3}
$$

Moreover, we have

$$
\Gamma_{00}^{k}=\Gamma_{k 0}^{0}=\Gamma_{00}^{0}=0, \quad \Gamma_{k l}^{i}=\frac{1}{2} \gamma^{i j}\left(\gamma_{j l, k}+\gamma_{j k, l}-\gamma_{k l, j}\right),
$$


and

$$
\Gamma_{k l}^{0}=\frac{1}{c} R \dot{R} \gamma_{k l}, \quad \Gamma_{0 l}^{k}=\frac{\dot{R}}{c R} \delta_{l}^{k},
$$

where $\gamma_{i k}$ is the three-metric associated with (25) and $\dot{R}=d R / d t$. The massive spin connection components $V_{\sigma}=\left(\Omega_{\sigma}\right)^{0 i}$ are determined in our Newtonian FRW manifold by

$$
\left(\Omega_{0}\right)^{i 0}=\left(\Omega_{0}\right)^{0 i}=0, \quad\left(\Omega_{k}\right)^{i 0}=e_{k}^{i} \frac{\dot{R}}{c R} .
$$

Since $G^{\mu \nu}$ satisfies the Bianchi identities:

$$
G_{; \nu}^{\mu \nu}=0
$$

we find from (33) that

$$
T_{; \nu}^{\mu \nu}=-\left(K^{\mu \nu}+H^{\mu \nu}\right)_{; \nu},
$$

where we have used $g_{; \nu}^{\mu \nu}=0$. In the unbroken phase of spacetime, we regain the standard energy-momentum conservation laws $\left(K^{\mu \nu}=0\right)$ :

$$
T_{; \nu}^{\mu \nu}=C_{; \nu}^{\mu \nu}=0 .
$$

\section{Superluminary Universe}

Let us consider a locally flat patch of space in the broken symmetry phase for $t<t_{c}$, with the line element:

$$
d s^{2}=c_{0}^{2} d t^{2}-\left(d x^{1}\right)^{2}-\left(d x^{2}\right)^{2}-\left(d x^{3}\right)^{2},
$$

where $c_{0}$ is the value of the velocity of light in the broken symmetry phase when $<\phi>\neq 0$. From the geodesic solution for light travel, $d s^{2}=0$, we get

$$
d t^{2}=\frac{1}{c_{0}^{2}}\left[\left(d x^{1}\right)^{2}+\left(d x^{2}\right)^{2}+\left(d x^{3}\right)^{2}\right] .
$$

In the limit $c_{0} \rightarrow \infty, d t \rightarrow 0$ and the Minkowski light cone is squashed. This solves the horizon problem, since all the points in an expanding bubble near the beginning of the Universe will be in communication with one another.

The horizon scale is determined by

$$
d_{H}(t)=c_{0} R(t) \int_{0}^{t} \frac{d t^{\prime}}{R\left(t^{\prime}\right)} .
$$

For $t>t_{c}$, this will have the usual value: $d_{H}(t)=2 c t$, since $R(t) \propto t^{1 / 2}$ for a radiation dominated Universe. Let us assume that for $t \leq t_{c}$, the speed of light is very large. During a first order phase transition, the velocity of light is assumed to undergo a discontinuous change from, for example, the value:

$$
c_{0} \sim 5 \times 10^{28} c
$$


for $t \leq t_{c}$ to $c_{0}=c$ (c is the present value of the velocity of light) for $t>t_{c}$, as shown in Fig. 1a. Then, we get for $t \leq t_{c}$ :

$$
d_{H}(t) \approx c_{0} g(t)
$$

where $g(t)$ is the functional time dependence arising from $R(t)$ in (48). Thus, for a fraction of time near the beginning of the Universe, all points of the expanding space will have been in communication with one another, solving the horizon problem.

Suppose the Higgs field that breaks the spacetime symmetries is characterized by a correlation length $\xi$. Then, the monopole density is approximately given by

$$
n_{M} \approx \xi^{-3}
$$

In the superluminary model, the bound on the length $\xi$ is given by

$$
\xi<d_{H}(t) \approx c_{0} g(t)
$$

so that the bound on the number density of monopoles is exponentially weakened. This solves the relic particle (monopole) problem.

For the standard FRW models the quantity $\Omega_{0}$, along with $H_{0}(H=\dot{R} / R)$, determines the cosmological model, for $\Omega_{0}$ and $H_{0}$ determine the radius of curvature:

$$
R^{2}=\left(c^{2} / H_{0}^{2}\right) /\left|\Omega_{0}-1\right|
$$

and the density: $\rho_{0}=\left(3 H_{0}^{2} / 8 \pi G\right) \Omega_{0}$. The present observational data restrict $\Omega_{0}$ to lie in the interval [0.01, few], which implies that $R \sim c / H_{0}$ and $\rho_{0} \sim \rho_{\text {crit }}$. Now consider in the unbroken phase the expression [2]:

$$
\Omega(t)=1 /[1-x(t)]
$$

where

$$
x(t)=\left(k c^{2} / R^{2}\right) /(8 \pi G \rho / 3)
$$

Then, we have

$$
\left|\Omega\left(10^{-43} \mathrm{sec}\right)-1\right| \leq O\left(10^{-60}\right)
$$

and

$$
|\Omega(1 \mathrm{sec})-1| \sim O\left(10^{-16}\right)
$$

Thus, the standard FRW cosmology is characterized by the very special initial data:

$$
|\Omega-1| \leq O\left(10^{-60}\right)
$$

This is the source of the flatness problem.

Let us assume, as an example, that in the spontaneously broken phase for $t_{c} \approx$ $10^{-35} \mathrm{~s}$, the velocity of light has the large value (49). The initial data at the Planck epoch are now:

$$
|\Omega-1| \leq O\left(10^{-3}\right)
$$


and

$$
\left|\left(k c_{0}^{2} / R^{2}\right)\right| /(8 \pi G \rho / 3) \sim O\left(10^{-3}\right),
$$

which implies much less fine tuning than in the standard FRW model of the Universe. Clearly, the value of $c_{0}$ is bounded by $c_{0}<10^{30} c$ to prevent an early collapse of the Universe.

We observe that in contrast to the inflationary model (which predicts that $\Omega=$ 1 ), the prediction for the value of $\Omega$ in the superluminary model depends on the detailed dynamics of the theory. Indeed, if we were to assume the equation of state: $\rho=$ const. in the broken phase for which $R(t)$ has the inflationary behavior: $R(t) \propto \exp (H t)$, then we would regain the standard inflationary prediction $\Omega=1$.

\section{Quantum Fluctuations}

We can allow the possibility in our model for generating the seed perturbations that can grow to form the large-scale structures. In the FRW model with $R(t) \propto t^{1 / 2}$, the physical wavelengths, which grow as $\lambda \propto R(t) \propto t^{1 / 2}$, will be far larger than the horizon (which grows as $c H(t)^{-1} \sim c t$ ) in the early phases. The comoving scales $\lambda$ cross the horizon only once. They start larger than the horizon, i.e. $\lambda \gg c H^{-1}$, and then cross inside the horizon at a later epoch. During the superluminary phase for $t<t_{c}$, the fluctuation wavelengths grow as: $\lambda \propto R(t)$. However, the horizon grows rapidly, $d_{H} \approx c_{0} g(t)$, where $c_{0}$ is given by (49), and will become equal to the physical wavelength at some time $t=t_{\text {exit }}$, after which it becomes larger than $\lambda(k)$ for a mode labeled by a wave vector $\vec{k}$.

After the symmetry is restored at $t>t_{c}$, the proper length $R(t)$ grows as $t^{1 / 2}$, whereas the horizon will increase as $c H(t)^{-1} \sim c t$. Therefore, the wavelength will be completely within the Hubble radius for an interval of time $\Delta t$. Thus, in the superluminary model the fluctuations are in microcausal connection very early in the Universe $\left(t \sim 10^{-35} \mathrm{~s}\right)$ and have time to grow into physical modes sufficiently large to form Galaxy structures. These fluctuations will have a gaussian form, provided any self-couplings of the matter fields are small.

Quantum fluctuations in matter fields taking place in the epoch, $t<t_{c}$, are candidates for seed perturbations. For example, the fluctuations associated with the Higgs field, $h$, which are responsible for breaking the spacetime symmetries could be such a candidate, satisfying the equation:

$$
\ddot{h}+3 H \dot{h}+\Gamma_{h} \dot{h}+V^{\prime}(h)=0,
$$

where $V(h)$ is given by $(31), \Gamma_{\phi}$ is the decay width associated with the decay of the $h$ particle and $V^{\prime}(h)=d V(h) / d h$.

In inflationary models, the Hubble parameter is constant during inflation, so all interesting scales begin sub-horizon, cross outside the Hubble radius during inflation, and at a later epoch cross back inside the horizon (at the usual time for standard 
FRW models). As a result, the spectrum of fluctuations predicted is close to a scale invariant spectrum-the Harrison-Zel'dovich spectrum 16. If we assume in the superluminary model that $c$ undergoes a discontinuous change, during a first order phase transition at $t \sim t_{c}$, to the value $c_{0}$ given by (49), then the horizon, $d_{H}(t)$, determined by (48) will also have a discontinuity in its first derivative with respect to $t$, and $d_{H}(t)$ for $t<t_{c}$ can be matched to $d_{H}(t)$ for $t \geq t_{c}$ in such a way that $\lambda$ crosses $d_{H}(t)$ twice, as in the inflationary model. The fluctuations are "frozen in" and leave an imprint on the metric tensor.

With the assumption that $h$ is spatially homogeneous, $H^{\mu \nu}$ takes the form of a perfect fluid, with the energy density and pressure given by

$$
\rho_{h}=\frac{1}{2} \dot{h}^{2}+V(h), \quad c^{2} p_{h}=\frac{1}{2} \dot{h}^{2}-V(h) .
$$

The fluctuations of the field $h$ are defined by the Fourier transform:

$$
\delta h_{k}=\int d^{3} x \exp (i \vec{k} \cdot \vec{x}) \delta h(x)
$$

and we have

$$
(\Delta h)_{k}^{2}=V^{-1} k^{3}\left|\delta h_{k}\right|^{2} / 2 \pi^{2}=\left(\frac{H}{2 \pi}\right)^{2} .
$$

Fluctuations in $h$ give rise to perturbations in the energy density:

$$
\delta \rho_{h}=\delta h\left(\frac{\partial V}{\partial h}\right)
$$

At horizon crossings, $\lambda_{\text {phys }} \sim c H^{-1}$, the gauge invariant quantity $\zeta$ takes the simple form $\zeta=\delta \rho /\left(\rho+c^{2} p\right)$ [17]. In the radiation dominated era and in the matter dominated era, $\zeta$ at horizon crossing is, up to a factor of order unity, equal to $\delta \rho / \rho$. Equating the values of $\zeta$ at the two horizon crossings, we find

$$
\left(\frac{\delta \rho}{\rho}\right)_{\mathrm{Hor}} \sim \frac{\delta h V^{\prime}}{\dot{h}^{2}} \sim \frac{H V^{\prime}}{2 \pi \dot{h}^{2}},
$$

where we have used the fact that $\delta h \sim H / 2 \pi$. We must now model $V^{\prime}$ and $\dot{h}$ at the phase transition, in order to estimate the density fluctuation, $\delta \rho / \rho$. Clearly, in contrast to the inflationary models, $H$ is rapidly varying at the phase transition. We have

$$
\dot{h} \sim \frac{H}{2 \pi \delta t} .
$$

A natural time scale for the duration of the phase transition is given by

$$
\delta t \sim\left(\frac{H}{2 \pi h^{3}}\right)^{1 / 2}
$$

Thus, if we choose $1 / H \sim 10^{-34}$ s and $h \sim M_{P} \sim 10^{43} s^{-1}$, then the duration of the phase transition is $\delta t \sim 10^{-48} \mathrm{~s}$. 
By assuming that $V(h)$, in (31), is dominated by $V(h) \sim \frac{\lambda}{4} h^{4}$, we obtain from (64) the scale invariant prediction for the amplitude:

$$
\left(\frac{\delta \rho}{\rho}\right)_{\text {Hor }} \sim \lambda
$$

We can fit the data measured by the Cosmic Background Explorer (COBE), which is consistent with a gaussian, scale invariant spectrum [18], by choosing the coupling constant $\lambda \sim 10^{-5}$, and using $\Delta T / T \sim \frac{1}{3} \delta \rho / \rho$. The measurements are quoted in terms of a spectral index $n$, with $n=1.1 \pm 0.5$. These measurements are also consistent with the predictions of inflationary models, and with other mechanisms of inhomogeneity generation, such as cosmic strings.

In standard inflationary models, the COBE data constrains the coupling constant of the inflaton models to be, $\lambda \sim 10^{-12}-10^{-14}$, which seems unnaturally small for the physical models considered in inflationary scenarios.

Another interesting feature of the superluminary model is that the Planck length, $L_{P}=\left(\hbar G / c_{0}^{3}\right)^{1 / 2} \rightarrow 0$ as $c_{0} \rightarrow \infty$ or, alternatively, the Planck mass, $M_{P}=$ $\left(\hbar c_{0} / G\right)^{1 / 2} \rightarrow \infty$ in this limit. This could have important implications for quantum fluctuations and for quantum gravity in the symmetry broken phase.

The results obtained above suggest that the superluminary model could be an attractive alternative to inflation as a solution to the initial value problem in cosmology.

\section{Acknowledgments}

I thank M. Clayton, N. Cornish, P. Savaria, G. Starkman and D. Tatarski for helpful and stimulating discussions. This work was supported by the Natural Sciences and Engineering Research Council of Canada.

\section{References}

[1] A. H. Guth, Phys. Rev. D23, 347 (1981).

[2] For reviews of inflationary cosmologies, see: E. W. Kolb and M. S. Turner, The Early Universe, Addison-Wesley Pub. Co. California, 1990; A. D. Linde, Rep. Prog. Phys. 47, 925 (1984).

[3] J. W. Moffat, Foundations of Physics 23, No. 3, 411 (1993)

[4] J. J. Halliwell, Conceptual Problems in Quantum Cosmology, eds. A. Ashtekar and J. Stachel, Birkhäuser, Boston, p.204, 1991. 
[5] L. D. Landau and E. M. Lifshitz, Statistical Physics, translated by J. B. Sykes and M. J. Kearsley, Addison-Wesley Publishing Company, Mass. p.427.

[6] S. Weinberg, Phys. Rev. D9, 3320 (1974).

[7] R. Mohapatra and G. Senjanovic, Phys. Rev. D20, 3390 (1979).

[8] P. Langacker and So-Young Pi, Phys. Rev. Letts. 45, 1 (1980).

[9] V. Kuzmin, M. Shaposhnikov, and I. Tkachev, Nucl. Phys. B196, 29 (1982).

[10] T. W. Kephart, T. J. Weiler, and T. C. Yuan, Nucl. Phys. B330, 705 (1990).

[11] S. Dodelson and L. M. Widrow, Phys. Rev. D42, 326 (1990).

[12] P. Salomonson and B. K. Skagerstam, Phys. Letts. B155, 98 (1985).

[13] C. Becchi, A. Rouet, and R. Stora, Ann. Phys. 98, 287 (1976).

[14] F. Halzen and A. D. Martin, Quarks and Leptons: An Introductory Course in Modern Particle Physics, John Wiley and Sons, New York, 1984.

[15] W. Rindler, Essential Relativity, 2nd ed. Springer-Verlag, New York, p. 223, 1977.

[16] E. R. Harrison, Phys. Rev. D1, 2726 (1970); Ya. B. Zel'dovich, Mon. Not. Roy. Astron. Soc. 160, 1p (1972).

[17] J. M. Bardeen, P. J. Steinhardt and M. S. Turner, Phys. Rev. D28, 679 (1983).

[18] G. F. Smoot et al., Ap. J. Letts. 396, L1 (1992); C. Bennett et al., ibid. 396, L7 (1992). 